\title{
Development of a Novel Method for the Preparation of Dithioacetal in the Presence of Titanium(IV) Chloride/Zinc in Dimethoxymethane
}

\author{
Ji-Hyun Lee, Hyung Jae Jeong, Chung Keun Jin, Sang Hun Jang, Min Kyu Kim, Yong-Jin Yoon,", and Sang-Gyeong Lee \\ Department of Chemistry and Research Institute of Life Science, GyeongSang National University, Jinju 660-701, Korea \\ *E-mail: leesang@nongae.gsnu.ac.kr \\ 'Department of Chemistry and Research Institute of Basic Science, GyeongSang National University, Jinju 660-701, Korea \\ Received October 25, 2004
}

Key Words : Low-valent titanium, Dithioacetal, Lewis acid, Methylene insertion

\begin{abstract}
Organosulfur compounds are important intermediates in organic synthesis. Since the pioneering report by Corey and Seebach, ${ }^{1}$ sulfur-stabilized carbanion has played an important role in organic synthesis. Recently, a new reaction method was developed that consists of a reductive lithiation of alkyl or arylthioesters ${ }^{2}$ and ring closing metathesis of titanium carbene complex prepared from thioacetals. ${ }^{3}$ In general, bis(methylthio)methane is used for the synthesis of ketene thioacetal ${ }^{4}$ by the reaction with aldehyde or ketone, and bis(phenylthio)methane is used for the synthesis of alkenes, ${ }^{5}$ ketones, ${ }^{6}$ allyic alchols ${ }^{7}$ and 1,3-dienes. ${ }^{8}$ In these reactions, only a few reagents, such as bis(methylthio)methane and bis(phenylthio)methane, are used presumably because the reagents are commercially available and can be prepared under limited conditions. In spite of the importance of dithioacetals or sulfur-stabilized carbanions in organic synthetic chemistry, only a few synthetic methods were known.
\end{abstract}

A general method for the preparation of bis(alkyl or arylthio)methanes is shown in Eq. 1. Sodium thioalkoxide or thiophenoxide reacts with diiodomethane in an appropriate solvent. ${ }^{6 \mathrm{~b}, 9}$

$$
\begin{aligned}
& \mathrm{RS}^{-} \mathrm{Na}^{+}+\mathrm{CH}_{2} \mathrm{I}_{2} \stackrel{\text { Solvent }}{\longrightarrow} \mathrm{RS}-\mathrm{CH}_{2}-\mathrm{SR} \\
& \mathrm{R}=\text { Methyl, Phenyl }
\end{aligned}
$$

For the preparation of dithioacetals by Eq. 1, the starting materials (alkyl or arylthiol) without a metal sensitive functional groups, such as hydroxy, carboxylic acid, and amino group, should be used. Therefore, only a few kinds of bis(alkyl or arylthio)methane can be prepared, and the poor supply of starting materials leads to the limited application in organic synthesis.

From this point of view, we need to develop a new method for the preparation of various dithioacetals. The preparation of this new class of various dithioacetals offers rich potential in synthetic organic chemistry. For example, the formation of the $\mathrm{S}_{-} \mathrm{CH}_{2}-\mathrm{S}$ bond between two N-CBZ-protected cysteins, which are of great interest for their biological activity, has been the object of synthesis of the methylenedithioacetal analogue of enkephalin, ${ }^{10}$ and intensive studies have been focused on the diastereoselective reactions employing dithioacetal as chiral auxiliaries. ${ }^{11}$

\section{Results and Discussion}

Recently, we developed titanium-mediated protection of heterocyclic thiols via the formation of MOM-, EOM- ethers without using a carcinogenic reagent, such as chloromethyl methyl ether. ${ }^{12}$ In continued research on the reaction between thiols and dimethoxymethane (DMM) or diethoxymethane (DEM), we found that the different products are obtained according to the nature of thiols. In the case of the reaction between heterocyclic thiols and DMM or DEM, only the corresponding MOM protected thioethers were obtained. The reaction between aromatic- and aliphatic thiols and DMM or DEM, however, gave the corresponding dithioacetal compounds (Eq. 2).

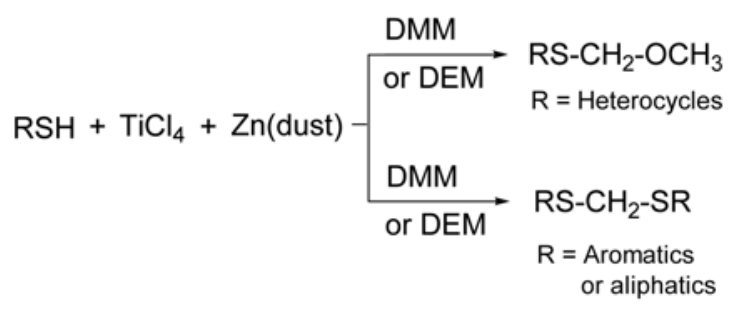

The results are summarized in Table 1 . The starting thiols containing ether (entry 3), ester (entry 2, 4, 6) and amino (entry 6) functional group were reacted under our conditions to give the corresponding dithioacetals in moderate yields. $\mathrm{N}$-CBZ-protected cystein 6 efficiently, providing the corresponding dithioacetal 18 in $76 \%$ yield (Entry 6). When dithiols, such as 7, 8 and 9 reacted with DMM or DEM in the same conditions, cyclic dithioacetals $\mathbf{2 0}, \mathbf{2 1}$ and $\mathbf{2 2}$ were obtained in good yields (Entry 7, 8 and 9). The commercial products $\mathbf{2 0}$ and $\mathbf{2 1}$ can be easily prepared in laboratory by using our simple method. Particularly, we obtained the macrocyclic compound 22 from the starting material $\mathbf{9}$. We investigated the methylene insertion reaction between thiol and oxygen atom for $\mathbf{1 0}$ (Entry 10). Our results show that the methylene insertion reaction between thiol and oxygen afforded only the corresponding macrocycle $\mathbf{2 3}$ in good yields (entry 8,9 ).

The analytical data $\left({ }^{1} \mathrm{H}\right.$ and $\left.{ }^{13} \mathrm{C}-\mathrm{NMR}, \mathrm{mp}\right)$ of the known products (compound $\mathbf{1 1}$ and 19) were matched with those of authentic samples and the new products were characterized 
Table 1. The Yield of RS-CH 2 -SR Obtained From The Reaction of R-SH and $\mathrm{TiCl}_{4} / \mathrm{Zn}$ in DMM

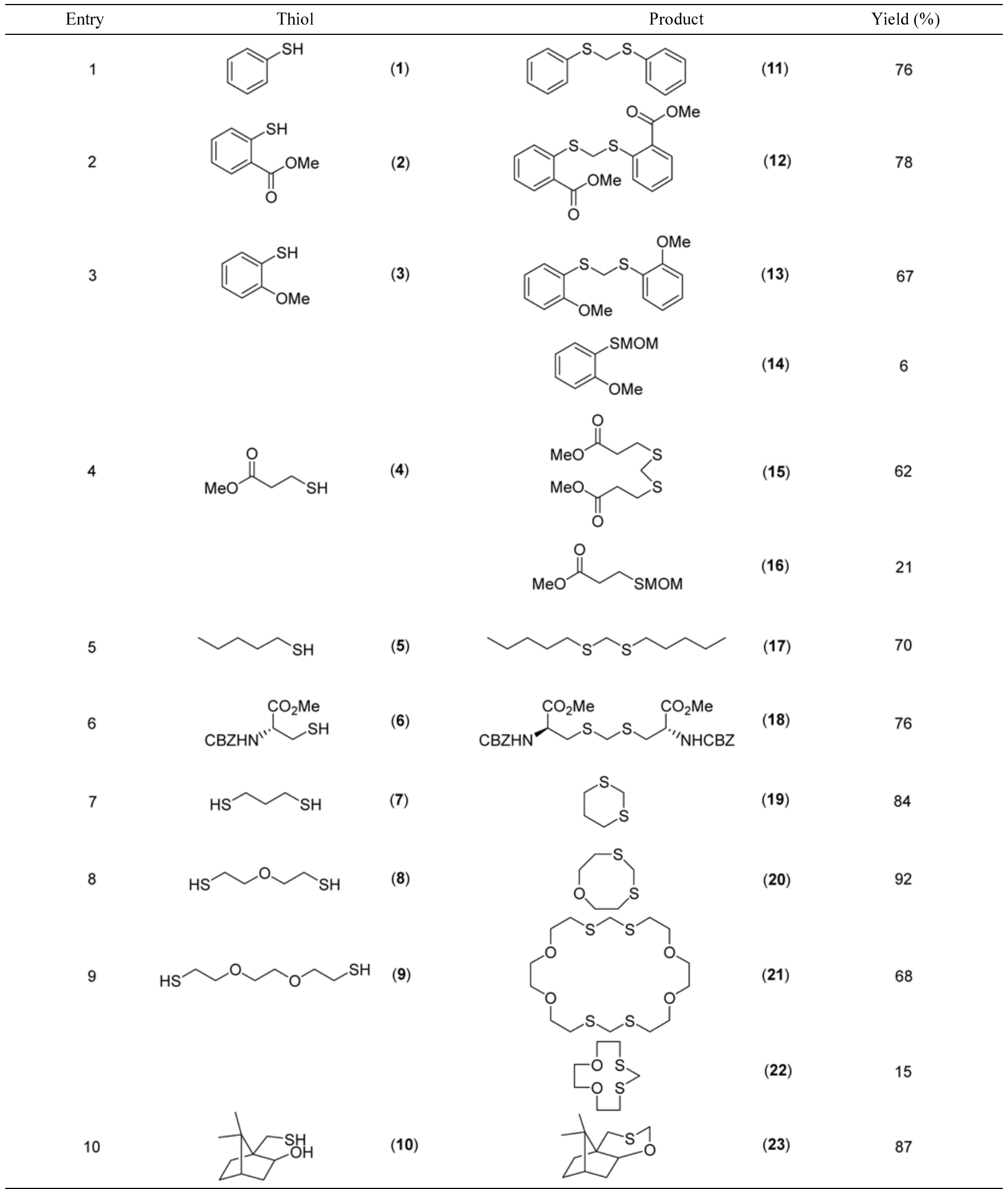

1) All reaction carried out under the same conditions. 2) Yield was calculated after purification.

by ${ }^{1} \mathrm{H}$ and ${ }^{13} \mathrm{C}$-NMR spectroscopy and mass spectrometry. Under the conditions described above, it was possible for $\mathrm{TiCl}_{4}$ to serve as a Lewis acid for the methylene insertion reaction of thiols. To test this possibility, reactions of thiols with dimethoxymethane in the presence of 4 equivalents of Lewis acids, such as $\mathrm{TiCl}_{4}, \mathrm{SnCl}_{4}$ and $\mathrm{AlCl}_{3}$, were investigated. Under these conditions, the reactions resulted in less than $30 \%$ conversion of thiol to dithioactal derivatives in 
$3 \mathrm{hr}$. The rate order to synthesize dithioacetals in the presence of Lewis acids was $\mathrm{TiCl}_{4} / \mathrm{Zn}[\mathrm{Ti}]>\mathrm{AlCl}_{3}>\mathrm{TiCl}_{4}>\mathrm{SnCl}_{4}$. Therefore, methylene insertion reaction under the $\mathrm{TiCl}_{4} / \mathrm{Zn}$ system appeared not simply as a $\mathrm{TiCl}_{4}$ catalyzed dithioacetal formation process but proceeded by another mechanism as a single electron transfer. ${ }^{13}$

We have developed a new synthetic method for the preparation of dithioacetal, which employs $\mathrm{TiCl}_{4} / \mathrm{Zn}$ in DMM. Dithioacetals containing versatile functional groups, such as ether, ester, and amino group can be obtained cleanly. Investigations on applying this thioacetals and the mechanistic aspects are currently underway and will be reported in due course.

\section{Experimental Section}

General remarks. All non-aqueous reactions were carried out under nitrogen. THF was distilled from $\mathrm{Na}$ / benzophenone; methanol was distilled from $\mathrm{Mg}$; methylene chloride was distilled from $\mathrm{CaH}_{2}$. Melting points were determined by an electrothermal digital melting point apparatus IA 9000 and uncorrected. NMR spectra were measured on a Bruker ARX-300 (500 MHz) spectrometer in $\mathrm{CDCl}_{3}$ solution used as an internal standard unless otherwise noted (value in ppm); coupling constants are reported in Hz. IR spectra were taken on a Hitachi $270-50 \mathrm{FT} / \mathrm{IR}$ spectrophotometer $\left(v_{\max }, \mathrm{cm}^{-1}\right)$. The elemental analysis was performed with LECO Micro Carbon Hydrogen Determinator (CHN-800). Mass spectra were obtained by using JEOL JMS-700 spectrophotometer. TLC was run on Merck precoated silicalgel plates. Merck silicagel 60 (230-400 mesh) was used for column chromatography.

A typical procedure for preparation of dithioacetals by thiols with $\mathrm{TiCl}_{4} / \mathrm{Zn}$ system. To a pre-stirred $\left(1 \mathrm{hr}, 0{ }^{\circ} \mathrm{C}\right)$ mixture of $\mathrm{TiCl}_{4}$ and zinc dust in a $1: 2$ molar equivalent ratio in $30 \mathrm{~mL}$ of dimethoxymethane was added the thiol (30 $\mathrm{mmol})$. The resulting solution was stirred for $2 \mathrm{hr}$ at $0{ }^{\circ} \mathrm{C}$ to $25^{\circ} \mathrm{C}$, poured into water $(50 \mathrm{~mL})$, stirred for $1 \mathrm{hr}$ at $\mathrm{rt}$, and extracted with EtOAc $(30 \mathrm{~mL} \times 3)$. The extract was washed with water and brine, dried over $\mathrm{MgSO}_{4}$, and concentrated in vacuo. Silicagel column chromatography ( $n$-Hexane/EtOAc $=10 / 1, \mathrm{v} / \mathrm{v})$ of the residue provided the pure methylene inserted products between alkyl or arylthio moiety (see Table 1).

Bis(2-methoxy carbonyl mercaptophenyl)methane (12): IR (KBr disk) 3010, 2940, 2910, 1700, 1560, 1430, 1250, 1140; ${ }^{1} \mathrm{H}$ NMR $\left(500 \mathrm{MHz}, \mathrm{CDCl}_{3}\right) \delta 7.97-7.95$ (dd, $J$ $=7.81,1.46 \mathrm{~Hz}, 1 \mathrm{H}), 7.54-7.52(\mathrm{~m}, 1 \mathrm{H}), 7.50-7.46(\mathrm{~m}, 1 \mathrm{H})$, 7.23-7.20 (m, 1H), 4.41 (s, 1H, -SCH), 3.90 (s, 3H, $\left.-\mathrm{OCH}_{3}\right)$; ${ }^{13} \mathrm{C}$ NMR (125 MHz, $\left.\mathrm{CDCl}_{3}\right) \delta 166.8,140.0,132.5,131.2$, 128.4, 126.8, 124.8, 52.1, 35.7; EI-MS m/z (real intensity) $348\left(\mathrm{M}^{+}, 23\right), 181$ (100), 151 (83), 136 (27), 121 (44), 108 (39), 77 (31).

Bis(2-methoxy mercaptophenyl)methane (13): IR (KRS-5 disk) 3050, 2990, 2930, 2830, 1580, 1470, 1240, 1020, 740; ${ }^{1} \mathrm{H}$ NMR $\left(500 \mathrm{MHz}, \mathrm{CDCl}_{3}\right) \delta 7.42-7.40(\mathrm{dd}, J=$ 7.62, $1.64 \mathrm{~Hz}, 1 \mathrm{H}), 7.25-7.21(\mathrm{~m}, 1 \mathrm{H}), 6.93-6.90(\mathrm{~m}, 1 \mathrm{H})$,
6.85-6.83 (dd, $J=8.20,1.0 \mathrm{~Hz}, 1 \mathrm{H}), 4.35(\mathrm{~s}, 1 \mathrm{H}), 3.87(\mathrm{~s}$, $\left.3 \mathrm{H},-\mathrm{OCH}_{3}\right) ;{ }^{13} \mathrm{C} \mathrm{NMR}\left(125 \mathrm{MHz}, \mathrm{CDCl}_{3}\right) \delta 132.0,128.5$, 121.0, 110.7, 55.7, 36.5; EI-MS m/z (real intensity) $292\left(\mathrm{M}^{+}\right.$, 35), 153 (100), 138 (67), 109 (12), 84 (14), 77 (14), 65 (8), $51(7)$.

Methoxy S-methoxymethyl 2-mercaptobenzene (14): IR (KRS-5 disk) 3100, 2950, 2900, 1600, 1500, 1450, 1250, $1200 ;{ }^{1} \mathrm{H}$ NMR $\left(500 \mathrm{MHz}, \mathrm{CDCl}_{3}\right) \delta 7.53-7.51(\mathrm{dd}, 1 \mathrm{H}, J=$ $1.63 \mathrm{~Hz}, J=7.70 \mathrm{~Hz}), 7.23-7.20(\mathrm{~m}, 1 \mathrm{H}), 6.94-6.92(\mathrm{~m}, 1 \mathrm{H})$, 6.87-6.85 (dd, $1 \mathrm{H}, J=0.96 \mathrm{~Hz}, J=8.21 \mathrm{~Hz}), 4.97(\mathrm{~s}, 2 \mathrm{H})$, $3.88(\mathrm{~s}, 3 \mathrm{H}), 3.42(\mathrm{~s}, 3 \mathrm{H}) ;{ }^{13} \mathrm{C} \mathrm{NMR}\left(125 \mathrm{MHz}, \mathrm{CDCl}_{3}\right) \delta$ $157.5,131.1,127.9,123.6,121.3,110.7,76.0,56.1,55.8$. EI-MS $m / z$ (real intensity) $184\left(\mathrm{M}^{+}, 100\right), 168$ (8), 154 (38), 139 (17), 109 (17), 77 (15).

Bis(methyl 3-mercaptopropionate)methane (15): IR (KRS-5 disk) 2950, 1740, 1440, 1360, 1250, 1040; ${ }^{1} \mathrm{H}$ NMR $\left(500 \mathrm{MHz}, \mathrm{CDCl}_{3}\right) \delta 3.70\left(\mathrm{~s}, 4 \mathrm{H},-\mathrm{OCH}_{3}\right.$ and $\left.-\mathrm{SCH}\right), 2.90(\mathrm{t}$, $J=7.3 \mathrm{~Hz}, 2 \mathrm{H}), 2.37(\mathrm{t}, J=7.3 \mathrm{~Hz}, 2 \mathrm{H}) ;{ }^{13} \mathrm{C} \mathrm{NMR}(125$ $\left.\mathrm{MHz}, \mathrm{CDCl}_{3}\right) \delta 26.1,34.6,36.0,52.2$, 172.6; EI-MS m/z (real intensity) $252\left(\mathrm{M}^{+}, 25\right), 165$ (8), 133 (100), 119 (11), 87 (12), 55 (56).

S-Methoxymethyl methyl 3-mercaptopropionate (16): IR (KRS-5 disk) 2950, 2820, 1740, 1430, 1360, 1180, 1080; ${ }^{1} \mathrm{H}$ NMR $\left(500 \mathrm{MHz}, \mathrm{CDCl}_{3}\right) \delta 4.64\left(\mathrm{~s}, 2 \mathrm{H},-\mathrm{SCH}_{2}\right), 3.70(\mathrm{~s}$, $\left.3 \mathrm{H},-\mathrm{OCH}_{3}\right), 3.34\left(\mathrm{~s}, 3 \mathrm{H},-\mathrm{OCH}_{3}\right), 2.86(\mathrm{t}, J=7.3 \mathrm{~Hz}, 2 \mathrm{H})$, $2.67(\mathrm{t}, J=7.3 \mathrm{~Hz}, 2 \mathrm{H}) ;{ }^{13} \mathrm{C} \mathrm{NMR}\left(125 \mathrm{MHz}, \mathrm{CDCl}_{3}\right) \delta$ $172.4,75.7,55.7,51.8,35.2,26.2$; EI-MS m/z (real intensity) $164\left(\mathrm{M}^{+}, 6\right), 149$ (100), 132 (33), 104 (15), 84 (35), 77 (21), 55 (25).

Bis(pentylthio)methane (17): IR (KRS-5 disk) 2930, 2960, 2860, 1460, 1380, 1200; ${ }^{1} \mathrm{H}$ NMR (300 MHz, $\left.\mathrm{CDCl}_{3}\right)$ $\delta 3.66(\mathrm{~s}, 2 \mathrm{H}), 2.62(\mathrm{t}, J=7.4 \mathrm{~Hz}, 4 \mathrm{H}), 1.61-1.57(\mathrm{~m}, 4 \mathrm{H})$, 1.39-1.30 (m, 4H), $0.90(\mathrm{t}, J=7.0 \mathrm{~Hz}, 6 \mathrm{H}) ;{ }^{13} \mathrm{C} \mathrm{NMR}(75$ $\left.\mathrm{MHz}, \mathrm{CDCl}_{3}\right) \delta 35.4,31.1,30.8,28.8,22.3$, 14.0; EI-MS $\mathrm{m} / \mathrm{z}$ (real intensity) $220\left(\mathrm{M}^{+}, 100\right) 117$ (40), 84 (44), 69 (41), 61 (39).

Bis(2-benzyloxycarbonylamino methyl 3-mercaptopropionate)methane (18): IR (KRS-5 disk) 3330, 3030, 2950, 1720, 1520, 1340, 1210, 1050; ${ }^{1} \mathrm{H}$ NMR (500 MHz, $\left.\mathrm{CDCl}_{3}\right)$ $\delta$ 7.34-7.25 (m, 5H), $5.65(\mathrm{~s}, 1 \mathrm{H},-\mathrm{NH}), 5.11(\mathrm{~s}, 2 \mathrm{H}$, $\left.-\mathrm{CH}_{2} \mathrm{Ph}\right), 4.60(\mathrm{~d}, J=5.25 \mathrm{~Hz}, 1 \mathrm{H}), 3.74$ (s, 3H, -OMe), 3.62 (s, 1H), 3.13-3.09 (m, 1H), 3.4-3.0 (m, 1H); ${ }^{13} \mathrm{C}$ NMR (125 $\left.\mathrm{MHz}, \mathrm{CDCl}_{3}\right) \delta 171.1,155.7,136.1,128.5,128.2,128.1$, 67.2, 53.4, 52.7, 36.5, 33.2; EI-MS m/z (real intensity) 550 $\left(\mathrm{M}^{+}, 78\right), 236$ (6), 207 (3), 162 (10), 146 (56), 91 (100), 79 (26), 59 (7).

[1,4,6] Oxadithiocane (20): IR (KRS-5 disk) 2910, 2850, 1460, 1400, 1290, 1210, 1100; ${ }^{1} \mathrm{H}$ NMR (500 MHz, $\mathrm{CDCl}_{3}$ ) $\delta 4.15\left(\mathrm{~s}, 2 \mathrm{H},-\mathrm{SCH}_{2}\right), 3.89(\mathrm{t}, J=4.79 \mathrm{~Hz}, 4 \mathrm{H}), 2.82(\mathrm{t}, J=$ $4.90 \mathrm{~Hz}, 4 \mathrm{H}) ;{ }^{13} \mathrm{C} \mathrm{NMR}\left(125 \mathrm{MHz}, \mathrm{CDCl}_{3}\right) \delta 73.2,38.7$, 31.2; EI-MS m/z (real intensity) $150\left(\mathrm{M}^{+}, 53\right), 136(7), 103$ (5), 84 (100), 78 (7), 61 (34), 51 (38).

1,9,12,20-Tetraoxa-4,6,15,17-tetrathiacyclodocosane (21): IR (KRS-5 disk) 2950, 2930, 1330, 1290, 1100; ${ }^{1} \mathrm{H}$ NMR (500 MHz, $\left.\mathrm{CDCl}_{3}\right) \delta 3.94(\mathrm{~s}, 1 \mathrm{H}), 3.74(\mathrm{t}, J=6.03$ $\mathrm{Hz}, 2 \mathrm{H}), 3.62(\mathrm{~s}, 2 \mathrm{H}), 2.85(\mathrm{t}, J=6.04 \mathrm{~Hz}, 2 \mathrm{H}) ;{ }^{13} \mathrm{C} \mathrm{NMR}$ $\left(125 \mathrm{MHz}, \mathrm{CDCl}_{3}\right) \delta 71.4,70.6,36.7,30.4 ;$ EI-MS m/z (real 
intensity) $388\left(\mathrm{M}^{+}, 25\right), 329$ (16), 315 (35), 207 (39), 194 (20), 147 (26), 103 (37), 75 (100), 61 (100), 60 (67).

1,9-Dioxa-4,6-dithiacycloundecane (22): IR (KRS-5 disk) 2960, 2910, 1120, 1100, 1050; ${ }^{1} \mathrm{H}$ NMR (500 MHz, $\left.\mathrm{CDCl}_{3}\right) \delta 4.07(\mathrm{~s}, 1 \mathrm{H}), 3.83(\mathrm{t}, J=5.83 \mathrm{~Hz}, 2 \mathrm{H}), 3.68(\mathrm{~s}$, $2 \mathrm{H}), 2.83(\mathrm{t}, J=5.83 \mathrm{~Hz}, 2 \mathrm{H}) ;{ }^{13} \mathrm{C} \mathrm{NMR}\left(125 \mathrm{MHz}, \mathrm{CDCl}_{3}\right)$ $\delta 73.6,71.8,36.3,31.2$; EI-MS $m / z$ (real intensity) $194\left(\mathrm{M}^{+}\right.$, 56), 149 (4), 135 (11), 117 (31), 103 (21), 91 (18), 75 (42), 61 (100), 60 (62).

11-Dimethyl-5-oxa-3-thiatricyclo[6.2.1.0 $\left.{ }^{1,6}\right]$ undecane (23): IR (KRS-5 disk) 2950, 2880, 1450, 1370, 1260, 1070, 970; ${ }^{1} \mathrm{H}$ NMR $\left(500 \mathrm{MHz}, \mathrm{CDCl}_{3}\right) \delta 4.78-4.75(\mathrm{dd}, J$ $=11.0,2.2 \mathrm{~Hz}, 1 \mathrm{H}), 4.72(\mathrm{~d}, J=10.9 \mathrm{~Hz}, 1 \mathrm{H}), 3.52-3.50$ $(\mathrm{dd}, J=8.0,3.2 \mathrm{~Hz}, 1 \mathrm{H}), 3.05$ (d, $J=14.1 \mathrm{~Hz}, 1 \mathrm{H}), 2.72-$ $2.68(\mathrm{dd}, J=14.1,2.2 \mathrm{~Hz}, 1 \mathrm{H}), 1.87-1.83(\mathrm{~m}, 1 \mathrm{H}), 1.72-$ $1.67(\mathrm{~m}, 1 \mathrm{H}), 1.46-1.43(\mathrm{~m}, 1 \mathrm{H}), 1.35(\mathrm{~s}, 3 \mathrm{H}), 1.03-1.01$ $(\mathrm{m}, 1 \mathrm{H}), 0.95-0.90(\mathrm{~m}, 1 \mathrm{H}), 0.91(\mathrm{~s}, 3 \mathrm{H}) ;{ }^{13} \mathrm{C}$ NMR $(125$ $\left.\mathrm{MHz}, \mathrm{CDCl}_{3}\right) \delta 84.6,77.3,77.1,76.8,69.38,46.7,45.5$, 43.1, 37.7, 34.9, 28.1, 27.3, 23.4, 20.4; EI-MS $m / z$ (real intensity) $198\left(\mathrm{M}^{+}, 78\right), 150$ (25), 135 (28), 121 (22), 108 (100), 93 (72), 67 (37), 55 (38).

\section{References}

1. Corey, E. J.; Seebach, D. Angew. Chem., Int. Ed. Engl. 1965, 4,
1075.

2. (a) Screttas, C. G.; Micha-Screttas, M. J. Org. Chem. 1978, 43, 1064. (b) Screttas, C. G.; Micha-Screttas, M. J. Org. Chem. 1979, 44, 713. (c) Cohen, T.; Bhupathy, M. Acc. Chem. Res. 1989, 22, 152.

3. Fujiwara, T.; Kato, Y.; Takada, T. Tetrahedron 2000, 56, 4859.

4. (a) Seebach, D.; Kolb, M.; Gröbel, B.-T. Ber. 1973, 106, 2277. (b) Seebach, D.; Bürstinghaus, R. Angew. Chem. Int. Ed. 1975, 14, 57. (c) Seebach, D.; Bürstinghaus, R. Synthesis 1975, 461.

5. Ager, D. J. Tetrahedron Lett. 1981, 22, 2932.

6. (a) Ager, D. J. Tetrahedron Lett. 1980, 21, 4763. (b) Corey, E. J.; Seebach, D. J. Org. Chem. 1966, 31, 4097. (c) Blatcher, P.; Grayson, J. I.; Warren, S. J. Chem. Soc. Chem. Commun. 1976, 547.

7. Foubelo, F.; Gutiérrez, A.; Yus, M. Tetrahedron Lett. 1999, 40, 8177.

8. Horikawa, Y.; Watanabe, M.; Fujiwara, T.; Takeda, T. J. Am. Chem. Soc. 1997, 119, 1127.

9. (a) Mosberg, H. I.; Omnaas, J. R. J. Am. Chem. Soc. 1985, 107, 2986. (b) Mosberg, H. I.; Omnaas, J. R.; Goldstein, A. Mol. Pharmacol. 1987, 31, 599.

10. Delogu, G.; Lucchi, O. D.; Maglioli, P. J. Org. Chem. 1991, 60, 4467.

11. (a) Ueki, M.; Shinozaki, K. Bull. Chem. Soc. Jpn. 1984, 57, 2156. (b) Ueki, M.; Ikeo, T.; Hokari, K.; Nakamura, K.; Saeki, A.; Komatsu, H. Bull. Chem. Soc. Jpn. 1999, 72, 829.

12. Jeong, H. J.; Yoon, E. Y.; Kim, M. K.; Lee, J. H.; Yoon, Y. J.; Lee, S. G. Bull. Korean Chem. Soc. 2003, 24, 1689.

13. Fürstner, A.; Hupperts, A.; Ptock, A.; Janssen, E. J. Org. Chem. 1994, 59, 5215. 